\title{
The migrant sensory neuritis of Wartenberg
}

\author{
WB MATTHEWS, MARGARET ESIRI \\ From the University Department of Clinical Neurology and Department of Neuropathology, The Radcliffe \\ Infirmary, Oxford, UK
}

SUMMARY Six cases are reported that conform to Wartenberg's description of migrant sensory neuritis. This is a benign relapsing and remitting condition in which pain and subsequent loss of sensation in the distribution of individual cutaneous nerves is induced by movement of the limbs inducing stretch. Sural nerve biopsy in one case showed loss of large myelinated fibres, axonal sprouting and some changes suggestive of ischaemia.

In 1958 Wartenberg ${ }^{\prime}$ collected his earlier brief descriptions and delineated a distinctive condition of migrant sensory neuritis. As in many original descriptions of disease, cases of probably quite different nature were included, but a syndrome of episodic, purely sensory symptoms, apparently produced by stretch of cutaneous nerves, was clearly described. It has since been almost entirely neglected, not being mentioned, for example, in Dyck et al's compendious volumes. ${ }^{2}$ Bruyn, ${ }^{3}$ in the Handbook of Clinical Neurology, presents no original observations but merely summarises Wartenberg's report. The syndrome is, however, not uncommon and we here describe six examples.

\section{Clinical description}

The essential feature is the sudden onset of a searing pain in the distribution of a cutaneous nerve, precipitated by a movement of a limb. The responsible movements are rapid but not extravagant, being banal activities that have often been performed previously without ill effect. Kneeling, stretching in bed, turning a handle or key or pointing the foot when putting on a sock are typical examples.

The pain is brief and, if the movement is immediately arrested, there are no after effects. If the movement is completed or is repeated within the succeeding days or weeks, cutaneous sensation is lost in the distribution of the affected nerve, but stretch is no longer painful. The patient who has experienced many such episodes comes to recognise the choice between a single severe pain followed by mildly inconvenient numbness, or repeated less severe

Address for reprint requests: Prof WB Matthews, The Radcliffe Infirmary, Oxford OX2 6HE, UK.

Received 1 July 1982 and in revised form 26 August 1982

Accepted 3 September 1982 pain, eventually, in an unguarded moment, culminating in a more severe attack and numbness. The relation to stretch is obvious to the patients, one remarking that: "the nerve feels too tight."

Recovery takes place over about six weeks but if there have been repeated episodes involving the same cutaneous nerve, slight subjective sensory change may persist in part of the affected area. Usually the movement that had induced the attack can afterwards be performed with impunity, at least for long periods. One of our patients had occasionally experienced a pain in the distribution of the second cervical root following some awkward movement of the neck. He remarked that this pain was different in character, in particular being less obviously superficial. Slight tingling persisted in the affected area for some hours only. It was thought that this symptom, a commonplace event, was unrelated and due to acute nerve root entrapment.

The cutaneous nerves known to have been involved in our patients are shown in table 1 . In some patients digital nerves were repeatedly affected in varying distribution and multiple attacks affecting the same nerve also occurred. The migrant character of the symptoms is extremely pronounced in some patients, attacks occurring in all four limbs over many years, often with long intervals of freedom followed by a number of attacks in quick succession. In other patients the condition appears to have subsided but follow up may not have been sufficient.

Both sexes are affected and the onset is in the fourth and fifth decades (table 2). Migrant sensory neuritis, although annoying, is benign and has a number of important negative characteristics. No motor involvement has been identified. The tendon reflexes are normal. There is no undue tendency to pressure palsies or, in our cases, to entrapment neuropathies. Occasionally pressure on a cutaneous nerve involved at the time can be painful, but Tinel's sign is uncommon. There is no nerve hypertrophy. General health is not disturbed and there is no association with diabetes or vascular disease. Routine haematology and ESR are normal. No family history of similar symptoms was obtained but relatives were not systematically examined.

Two illustrative cases are described: 
Table

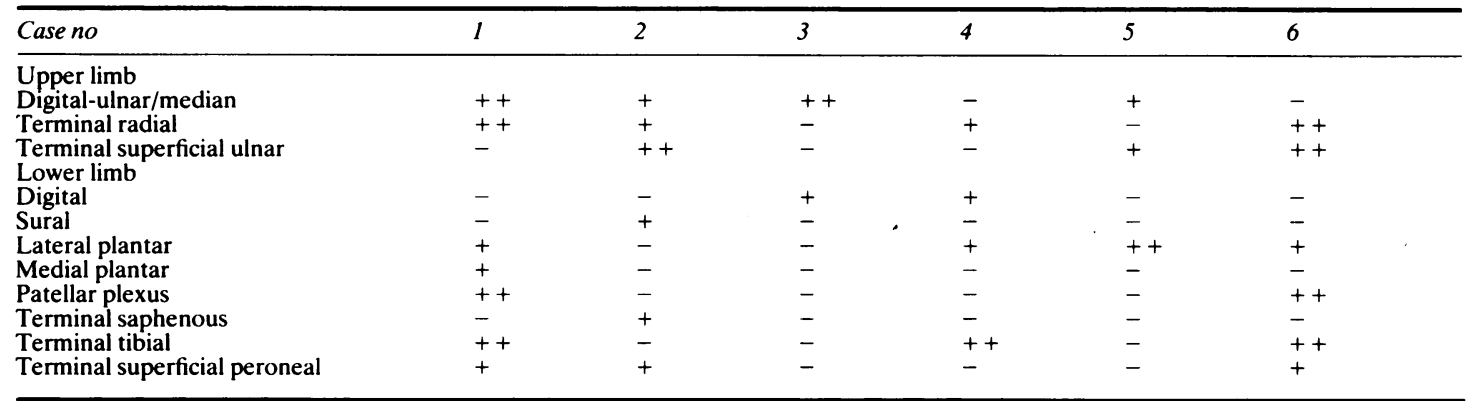

Cutaneous nerves known to be affected. $+=$ unilateral $;++=$ bilateral.

Table 2

\begin{tabular}{lllllll}
\hline Case no & 1 & 2 & 3 & 4 & 5 & 6 \\
\hline Sex & M & F & M & M & M & M \\
Age at onset (yr) & 30 & 34 & 41 & 32 & 44 & 32 \\
Present age (yr) & 62 & 38 & 44 & 38 & 49 & 42 \\
\hline
\end{tabular}

Case 1 A male physician, now aged $62 \mathrm{yr}$, otherwise in good health, believed that his symptoms began around the age of 30 . He had seldom paid serious attention to them, as being obviously related to the cutaneous nerves, and details of the onset can no longer be recalled. For many years the only areas involved were the terminal radial on both sides, and digital nerves in the hands, particularly those of the index finger. At first, the sequence of pain on stretch leading to numbness continued unchanged but after repeated episodes affecting the outer border of the left index finger slight alterations of sensation persisted in that area for many months to the time of reporting. Around the age of 55 cutaneous areas on the lower limbs began to be affected, of which the most troublesome were the patellar plexus on each side, at one time producing symmetrical patches of numbness between the patella and the head of the fibula. Kneeling while gardening was the usual cause of stretch and again sensory change, amounting to no more than slight loss of sensitivity to touch, became persistent. No specific investigations were carried out, the normal nerve conduction values mentioned in the text being obtained during neurophysiological experiments.

Case 2 A woman of $36 \mathrm{yr}$, first seen in May 1979, had suffered from intermittent low back pain and left sided sciatica since a fall from her bicycle at the age of 27 . When 34 , new symptoms developed. On plantar flexing the right foot she would experience sharp pain over the instep. After several such brief episodes she became numb in the same area and the movement could be made without pain. The numbness recovered in a month but she then had exactly the same sequence of symptoms in the right heel, the pain being induced by dorsiflexion. Pain and numbness recurred in the right foot and shortly before she was seen similar symptoms affected the inner border of the left fifth finger. The early symptoms had been attributed to a presumed prolapsed lumbar intervertebral disc but sensory loss in the hand led to a suspicion of multiple sclerosis both by the patient and the referring physician. When examined, the left ankle jerk was absent, no doubt owing to her sciatica. Loss of cutaneous sensation was found within the distribution of the right sural and terminal superficial peroneal nerves and of the digital nerve to the medial border of the left fifth finger. Six months later she reported several further episodes and there was sensory loss on the right heel, the lateral side of the right great toe, the outer side of the left little toe and the medial border of the left hand and fifth finger. She could remember that most of those had been preceded by sudden pain on movement but she could not recall precise details. After a further six months most of these areas of numbness had cleared but she had new symptoms in the terminal radial distribution on the left. Her sciatica was relieved by removal of a lumbar prolapsed disc but her other symptoms have continued, with repeated episodes affecting both hands and feet, as shown in table 1. She has become accustomed to her symptoms and no longer believes that she has multiple sclerosis.

\section{Investigations}

As the condition was plainly harmless investigation was not vigorously pursued. Motor and sensory conduction velocities in the main nerve trunks in the upper and lower limbs were repeatedly found to be normal in case 1 . The latencies of knee and ankle jerks and of the $\mathrm{H}$ reflex were also normal. A normal sensory nerve action potential was obtained from the terminal radial nerve that had previously been affected, although sensation was normal at the time of investigation. Case 6 (kindly referred by Dr JM Oxbury) was more thoroughly investigated. Dr G Rushworth found no EMG or motor conduction abnormality in the right upper limb, but the sensory nerve action potentials from the digital nerves of the right hand were all mildly reduced in amplitude. No potential could be recorded from the right sural nerve that had been clinically involved some months earlier. This nerve was biopsied.

Eight nerve fascicles were contained within the biopsy specimen. There was loss of large myelinated nerve fibres from all these fascicles though some were more severely affected than others (see histogram, fig 1). In the fascicle containing no large diameter fibres there was an increase in the proportion of small myelinated fibres and these were grouped in clusters producing a pattern suggestive of 


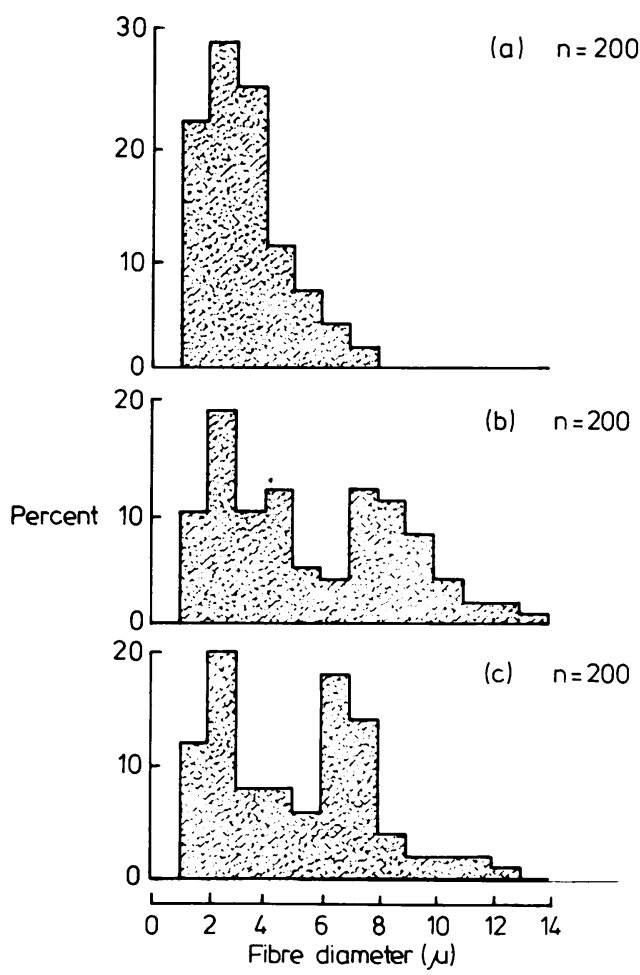

Fig 1 Histogram of myelinated nerve fibre diameters in three different fascicles within the nerve biopsy from Case 6. Loss of large diameter fibres and an increase in the proportion of small diameter fibres is particularly evident in fascicle $a$.

regeneration with axonal sprouting. Collagen was moderately increased in all compartments of the nerve. Elastic fibres were present in normal numbers. In longitudinal sections of the nerve there were focal areas of endoneurial oedema with splaying apart of nerve fibres and loss of myelin, and, in the same areas, increase in acid mucopolysaccharide detectable with an Alcian blue stain. Some abnormalities were observed in small perineurial and epineurial blood vessels with occlusion of one small vessel by loose connective tissue (fig 2 ) and at another level, the presence of irregular vascular channels possibly representing a recanalised artery. There was no evidence of a vasculitis. The teased fibre preparation showed no segmental demyelination. Electron microscopy confirmed the presence of axonal sprouts (fig 3) and increase in endoneurial collagen, but showed no specific pathological changes. Unmyelinated fibres showed no abnormalities.

\section{Discussion}

In five of our six cases the relapsing and remitting sensory symptoms had suggested a diagnosis of

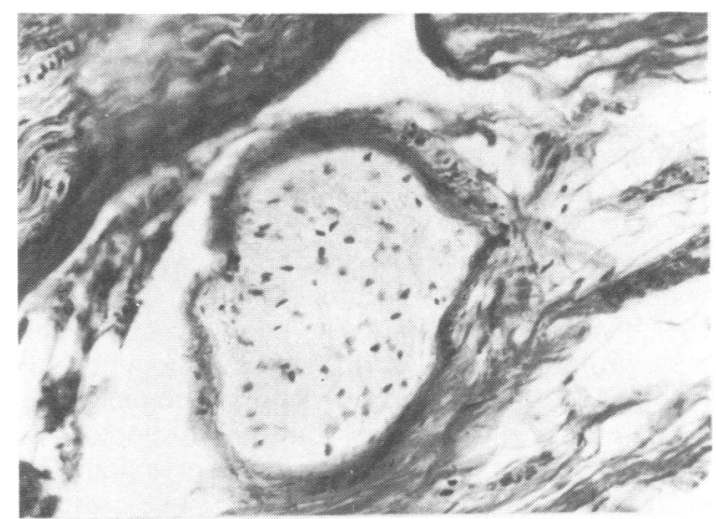

Fig 2 Case 6. Small perineural blood vessel occluded by organised thombus. $\times 125$

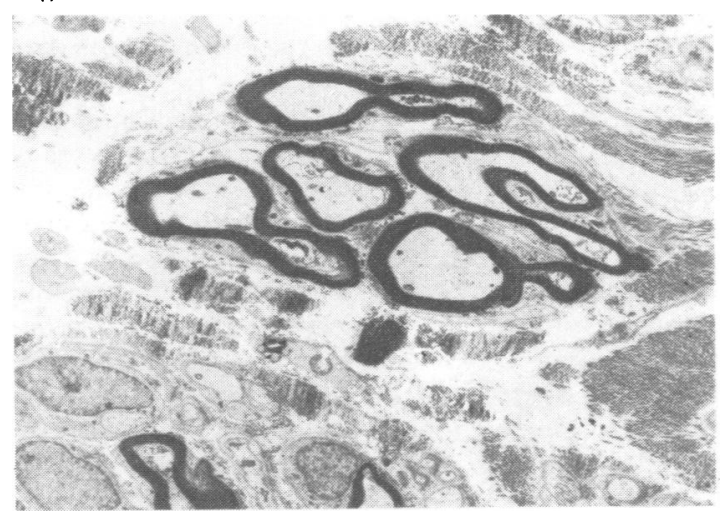

Fig 3 Case 6. Low power electron micrograph showing a cluster of small diameter myelinated nerve fibres indicative of axonal sprouting. $\times 2160$

multiple sclerosis, either to the patients or their medical advisers. Indeed this was probably the correct diagnosis in Wartenberg's' ${ }^{1}$ case 3 . The clinical features are, however, quite distinctive, and the sensory loss is clearly in the distribution of individual cutaneous nerves rather than segmental. None of our patients experienced numbness on the trunk or face, as described by Wartenberg. ' It is possible to accept as classical examples of his own syndrome only his cases 1 and 8 . The case history and clinical findings in the former were described in detail and closely resembled those of our patients. The latter, case 8, a man of $86 \mathrm{yr}$, gave a typical history extending over many years but was not examined. In his other six cases many of the symptoms appear to have been those of entrapment syndromes, or multiple symmetrical peripheral neuropathy, or were unexplained but not related to stretch. Wartenberg ${ }^{1}$ believed that Schultze $^{4}$ had described a single example of the same migrant neuritis, apparently in 
himself, but the description of the sensory symptoms, although extremely minute, bears little resemblance to that in our patients.

Asbury et $a l^{5}$ described a condition of sensory perineuritis. Their two patients presented with pain in the feet on prolonged standing. Sensory nerves were painful when tapped and Tinel's sign was. positive. Individual cutaneous nerves were involved in a relapsing and remitting fashion. No effect of stretch was remarked and the pain appears to have been prolonged. The condition was thought to be different from that described by Wartenberg. The most important distinction is in the histology of the nerve biopsies that showed chronic granulomatous lesions of the perineurium, in some respects reminiscent of leprosy. It appears to have been a true inflammatory neuritis and caused moderate disability.

Wartenberg commented on the effects of stretch in causing pain but did not emphasise the immediate subsequent sensory loss. The connection is so evident, especially to the patients, that it is a reasonable assumption that the fibre loss we observed was the result of stretching the nerve. The investigation of the effects of stretching has been largely confined to main nerve trunks, with particular regard to surgical repair of nerve injuries. All nervous tissue must be capable of sustaining unharmed some degree of stretch and of subsequent retraction. In peripheral nerve axons pursue an undulating course and straighten on being stretched. ${ }^{6}$ Stretch to the point of damage causes narrowing of the perineurial tubes and injury to myelin and axons. ${ }^{7}$ Retraction following physiological degrees of stretch may be due to the elastic properties of the perineurium or possibly to contractile elements. ${ }^{8}$

In migrant sensory neuritis axonal damage is sustained with normal degrees of stretch. Abnormal narrowing of the perineurial tube or anchoring of the nerve fibres to their connective tissue coverings can be suggested as possible mechanisms. The biopsy in our patient showed some increase in endoneurial collagen but threw no light on the possible effects of movement. The vascular changes observed were not those of arteritis but it is conceivable that the effect of stretch is on the immediate arterial supply to the nerve and that the symptoms arise in a nerve already affected by ischaemia. The pain itself can scarcely be directly due to ischaemia in view of its instantaneous onset with movement. None of our patients showed evidence of any vascular disease.

Our main purpose is to draw attention to the characteristic clinical course of this benign but often alarming condition.

\section{References}

' Wartenberg R. Neuritis. Sensory Neuritis. Neuralgia. New York: Oxford University Press, 1958:233-47.

${ }^{2}$ Dyck PJ, Thomas PK, Lambert EH, eds. Peripheral Neuropathy. Philadelphia: WB Saunders, 1975.

${ }^{3}$ Bruyn GW. Migrant sensory neuritis. In: Vinken PJ and Bruyn GW, eds. Handbook of Clinical Neurology, Vol 8. Amsterdam: North Holland, 1970:165-8.

${ }^{4}$ Schultze F. Akute disseminierte Hauthyperalgesie (multiple sensible neuritis?). Deutsche Zeitschrift fur Nervenheilkunde 1925;87:108-12.

${ }^{5}$ Asbury AK, Picard EH, Baringer JR. Sensory perineuritis. Arch Neurol 1972;26:302-12.

- Clarke E, Bearn JG. The spinal nerve bands of Fontana. Brain 1972;95:1-20.

${ }^{7}$ Haftek J. Stretch injury of peripheral nerve. Acute effects of stretching in rabbit nerve. J Bone Joint Surg 1970;52B:354-65.

${ }^{8}$ Ross MH, Reith EJ. Perineurium: evidence for contractile elements. Science 1969;165:604-5. 